\title{
SUPERPROCESSES IN RANDOM ENVIRONMENTS ${ }^{1}$
}

\author{
BY LEONID MYTNIK \\ Technion-Israel Institute of Technology
}

\begin{abstract}
We study the limiting behavior of large branching particle systems undergoing random motion, whose branching mechanism is affected by a random environment. The weak convergence result is established for a sequence of such particle systems and the limiting process is characterized as the unique solution of a martingale problem. The proof of uniqueness of the solution for the martingale problem requires an extension of standard duality techniques.
\end{abstract}

0. Introduction. Measure-valued branching Markov processes (or superprocesses) arise as limits of branching particle systems undergoing random migration and critical (or asymptotically critical) branching. There exist a number of constructions of superprocesses. As an example, consider the following system.

Let $Y$ be a Feller process in a locally compact Polish space $E$ with generator $(A, \mathscr{D}(A))$. Assume that, at time $t=0, K_{n} \geq 1\left(K_{n} \sim n\right)$ particles are located in $E$. Each of these $K_{n}$ particles follows the path of an independent copy of $Y$ until time $t=1 / n$. At time $1 / n$ each particle, independently of the others, either dies or splits into two, with probability $1 / 2$ for each event. The individual particles in the new population then follow the path of an independent copy of $Y$, starting at their place of birth, in the interval $[1 / n, 2 / n)$, and the pattern of alternating critical branching and spatial spreading continues until, with probability 1 , there are no particles left alive. Let $\tilde{X}_{t}^{n}(\cdot)$ be the measure-valued Markov process, defined as

$$
\tilde{X}_{t}^{n}(B)=\frac{\text { number of particles in } B \text { at time } t}{n},
$$

where $B \in \mathscr{B}(E)$ are the Borel sets in $E$. It is well known that, under mild conditions, $\left\{\tilde{X}_{t}^{n}\right\}$ converges, as $n \rightarrow \infty$, on an appropriate Skorohod space, to a measure-valued process known as the " $Y$ superprocess."

The characteristic feature of this construction is that the only interaction between the particles is that the time and place of birth of offspring coincide with the time and place of death of their parent.

In this article we investigate the limiting behavior of particle systems whose branching is affected by a random environment. In particular, we shall

\footnotetext{
Received June 1995; revised December 1995.

${ }^{1}$ Research supported in part by U.S.-Israel Science Foundation and Israel Academy of Sciences.

AMS 1991 subject classifications. Primary 60G57, 60F17; secondary 60J80, 60H15.

Key words and phrases. Measure-valued processes, superprocess, martingale problem, duality, random environment.
} 
assume that at birth/death times there exists a small correlation between the number of offspring at different points of the state space $E$. For example, let $\left\{\xi_{k}, k \in \mathbb{N}\right\}$ be a sequence of independent identically distributed mean-zero random fields on $E$ with finite third moment and covariance function $g(x, y)$. Denote by $\xi_{k}^{(n)}$ the truncated random field: $\xi_{k}^{(n)}(\cdot) \equiv \sqrt{n} \wedge\left(\xi_{k}(\cdot) \vee-\sqrt{n}\right)$. Assume in the previous model that, conditionally on $\xi_{k}^{(n)}$, each particle, independently of the others, at time $k / n$ splits into two with probability

$$
\frac{1}{2}+\frac{1}{2 \sqrt{n}} \xi_{k}^{(n)}(x)
$$

or dies with probability

$$
\frac{1}{2}-\frac{1}{2 \sqrt{n}} \xi_{k}^{(n)}(x),
$$

where $x$ is the location of the particle. Define the measure-valued process $X_{t}^{n}$ in the same way as in the previous construction. We shall study the limiting behavior of $\left\{X^{n}\right\}$ as $n \rightarrow \infty$, and we shall show that if $X_{0}^{n}$ converges to $m$, then $\left\{X^{n}\right\}$ converges to the measure-valued process $X$, which is the unique solution of the following martingale problem:

$$
\left\{\begin{array}{l}
\text { For all } \phi \in \mathscr{D}(A), \\
\quad Z_{t}(\phi) \equiv X_{t}(\phi)-m(\phi)-\int_{0}^{t} X_{s}(A \phi) d s \\
\text { is an } \mathscr{F}_{t}^{X} \text { with continuous square-integrable martingale } \\
Z_{0}(\phi)=0 \text { and } \\
\langle Z(\phi)\rangle_{t}=\int_{0}^{t} X_{s}\left(\phi^{2}\right) d s \\
\quad+\int_{0}^{t} \int_{E \times E} g(x, y) \phi(x) \phi(y) X_{s}(d x) X_{s}(d y) d s
\end{array}\right.
$$

where $\nu(\phi)$ denotes $\int \phi d \nu$ for any measure $\nu$.

The remainder of this article is organized as follows. Our proofs are based on the martingale problem approach and the basic ideas of this approach are discussed in Section 1. The proof of uniqueness of the solution for the martingale problem in our case requires an extension of standard duality techniques, which is also considered in this section. In Section 2 we survey some previously studied models of branching in a random environment, but without any spatial motion. The material in this section provides motivation for our own model. Section 3 is devoted to our model. We present the proofs in the last section.

1. Preliminaries: martingale problems. In this section we give a very brief introduction to martingale problems and introduce and prove a new duality result that we believe is of independent interest, beyond the specific application of the current paper. We start with some notation and definitions. Let $(E, d)$ be a metric space and let $B(E)[\operatorname{resp} . C(E), \bar{C}(E)]$ be the set of 
bounded (resp. continuous, bounded continuous) Borel-measurable functions on $E$. In general, if $F$ is a set of functions on $E$, write $F_{+}\left(F_{++}\right)$for nonnegative $\left[\left\{f \in F: \inf _{E} f(x)>0\right\}\right]$ functions in $F$. Denote by $\mathscr{B}(E)$ both the Borel $\sigma$-algebra and Borel-measurable functions on $E$ and by $\mathscr{P}(E)$ the set of Borel probability measures on $E$. Let $A$ be a subset (not necessary linear) of $B(E) \times B(E)$.

Definition 1.1. By a solution of the martingale problem for $A$ we mean a measurable stochastic process $X$ with values in $E$ defined on some probability space $(\Omega, \mathscr{F}, P)$, such that, for each $(f, g) \in A$, the process

$$
f(X(t))-\int_{0}^{t} g(X(s)) d s
$$

is a martingale with respect to the filtration

$$
\widetilde{\mathscr{F}_{t}^{X}}=\mathscr{F}_{t}^{X} \vee \sigma\left(\int_{0}^{t} h(X(u)) d u: s \leq t, h \in B(E)\right),
$$

where $\mathscr{F}_{t}^{X} \equiv \sigma(X(s): s \leq t)$.

When an initial distribution $\mu \in \mathscr{P}(E)$ is specified, we say that a solution of the martingale problem for $A$ is a solution of the martingale problem for $(A, \mu)$ if $P X(0)^{-1}=\mu$.

Note that if $X$ is right continuous, then $\widetilde{\mathscr{F}}_{t}^{X}=\mathscr{F}_{t}^{X}$.

DeFinition 1.2. We say that uniqueness holds for the solutions of the martingale problem for $(A, \mu)$ if any two solutions $X, Y$ have the same finite-dimensional distributions. That is, for every $m>0$ and all choices of $t_{k}, 0 \leq t_{1}<t_{2}<\cdots<t_{m}$, the following holds:

$$
\begin{aligned}
& P\left\{\left(X\left(t_{1}\right), \ldots, X\left(t_{m}\right)\right) \in \Gamma\right\} \\
& \quad=P\left\{\left(Y\left(t_{1}\right), \ldots, Y\left(t_{m}\right)\right) \in \Gamma\right\} \quad \forall \Gamma \in \mathscr{B}(E) \times \cdots \times \mathscr{B}(E) .
\end{aligned}
$$

The primary value of the martingale problem approach is that it allows easy characterization of the finite-dimensional distributions for a wide variety of stochastic processes. That is, if we know that, for some process $X$ and operator $A$, (1.1) holds and uniqueness for $(A, \mu)$ holds, then we have determined the finite-dimensional distributions of $X$.

It is well known that the task of establishing uniqueness for the martingale problem reduces to verifying that any two solutions have the same one-dimensional distributions (see, e.g., Theorem 4.4.2 of [7]). One approach to doing this involves the notion of duality, and this requires establishing the existence of another (dual) process (see, e.g., Chapter 4.4 of [7]). Careful examination of this approach shows that it is in fact unnecessary to prove the existence of the dual process, and it suffices to find some approximating sequence of processes that are not necessarily required to converge in a full function space sense. The following theorem captures this idea. 
THEOREM 1.3. Let $E_{1}$ be a complete, separable metric space and let $E_{2}$ be some topological space. Let $A_{1} \subset B\left(E_{1}\right) \times B\left(E_{1}\right), f \in \mathscr{B}\left(E_{1} \times E_{2}\right), \beta \in \mathscr{B}\left(E_{2}\right)$ and $\tilde{E}_{2} \subset E_{2}$. Suppose that $\left\{f(\cdot, y): y \in \tilde{E}_{2}\right\}$ is separating on $\mathscr{P}\left(E_{1}\right)$ and that, for every $\nu \in \mathscr{P}\left(E_{1}\right)$ with compact support and every $y \in \tilde{E}_{2}$, there exists a sequence of processes $\left\{Y^{(n)}\right\}$, taking values in $E_{2}$, such that $Y^{(n)}(0)=y$ and

$$
E[f(X(t), y)]=\lim _{n \rightarrow \infty} E\left[f\left(X(0), Y^{(n)}(t)\right) \exp \left\{\int_{0}^{t} \beta\left(Y^{(n)}(s)\right) d s\right\}\right]
$$

for every $t \geq 0$ and each solution $X$ of the martingale problem for $\left(A_{1}, \nu\right)$, independent of $\left\{Y^{(n)}\right\}$. Then for each $\mu \in \mathscr{P}\left(E_{1}\right)$ for uniqueness holds for the martingale problem for $\left(A_{1}, \mu\right)$.

Proof. The proof is analogous to that of Theorem 4.4.7 of [7]. Let $X$ and $\tilde{X}$ be solutions of $\left(A_{1}, \nu\right)$, where $\nu \in \mathscr{P}\left(E_{1}\right)$ has compact support, and choose arbitrary $y \in \tilde{E}_{2}$. Then, by our assumptions, there exists a sequence of processes $\left\{Y^{(n)}\right\}$ independent of $X, \tilde{X}$ taking values in $E_{2}$, such that $Y_{0}=y$ and

$$
\begin{aligned}
& E[f(X(t), y)] \\
& \quad=\lim _{n \rightarrow \infty} E\left[f\left(X(0), Y^{(n)}(t)\right) \exp \left\{\int_{0}^{t} \beta\left(Y^{(n)}(s)\right) d s\right\}\right] \\
& \quad=E[f(\tilde{X}(t), y)] .
\end{aligned}
$$

It is true for each $y \in \tilde{E}_{2}$; hence, any two solutions of the martingale problem for $\left(A_{1}, \nu\right)$ have the same one-dimensional distributions. Now, taking arbitrary $\nu \in \mathscr{P}\left(E_{1}\right)$ and continuing as in the proof of Theorem 4.4.7 of [7], we are done.

2. Branching process in random environments. Recently there has been growing interest in the study of systems in random media (environments) and systems with interactions. Some examples of the introduction of interactions and random media into the superprocess include:

1. Making the rate of branching be dependent on random catalysts (see [4]).

2. Introducing interactions in which the rate of branching, the branching law and the motion process are dependent on the state of the system of particles (see [15], [17] and [18]).

In our work the random medium will affect the branching mechanism. There has been substantial interest in such a model for the somewhat simpler case of particles moving on the discrete lattice $Z^{d}$, without going to the infinite density limit corresponding to the superprocess (e.g., [1], [9] and [8]).

To motivate our result, we recall the limiting behavior of a model closely related to ours but without the spatial motion. The result that we shall describe below was originally conjectured by Keiding [13] and proved by Helland [10]. In the form introduced here, it appeared in [14]. 
For each $n$ let $\left\{\left(\xi_{m}^{(n)}, X_{1, m}^{(n)}, X_{2, m}^{(n)}, \ldots\right)\right\}_{m=1}^{\infty}$ be a sequence of independent, identically distributed $\left(\mathbb{R} \times \mathbb{Z}_{+}^{\infty}\right)$-valued random variables, $X_{k, m}^{(n)} \geq 0$, and for fixed $m, n$ suppose $X_{1, m}^{(n)}, X_{2, m}^{(n)}, \ldots$ are conditionally independent and identically distributed given $\xi_{m}^{(n)}\left(\xi_{m}^{(n)}\right.$ determines the "environment"). We define a branching process $Z_{n}(\cdot)$, with initial state $Z_{n}(0)$, recursively by

$$
Z_{n}(m)=\sum_{i=1}^{Z_{n}(m-1)} X_{i, m-1}^{(n)} .
$$

Then $X_{k, m}^{(n)}$ is the number of offspring of the $k$ th member of the $m$ th generation and $Z_{n}(m)$ gives the number of offspring in the $m$ th generation.

Assume that $Z_{n}(0) / n \rightarrow x_{0}$ and define

Let

$$
X_{n}(t)=\frac{Z_{n}([n t])}{n} .
$$

and suppose that

$$
E\left[X_{k, m}^{(n)} \mid \xi_{m}^{(n)}\right]=M_{m}^{(n)}
$$

$$
\begin{aligned}
& \lim _{n \rightarrow \infty} n E\left[X_{k, m}^{(n)}-1\right]=\beta, \quad \lim _{n \rightarrow \infty} n E\left[\left(M_{m}^{(n)}-1\right)^{2}\right]=\gamma, \\
& \lim _{n \rightarrow \infty} E\left[\left(X_{k, m}^{(n)}-1\right)^{2}\right]=\sigma^{2}, \quad \sup _{n} E\left[\left(X_{k, m}^{(n)}-1\right)^{3}\right]<\infty .
\end{aligned}
$$

Let $\Rightarrow$ denote weak convergence. Then we have the following result.

Theorem 2.1 (Kurtz [14]). Let $X_{n}$ be as above. Then $X_{n} \Rightarrow X$, where $X$ is a diffusion whose generator is the closure of

$$
A=\left\{\left(f, \frac{1}{2}\left(\alpha x+\gamma x^{2}\right) f^{\prime \prime}+\beta x f^{\prime}\right): f \in C_{c}^{\infty}[0, \infty)\right\} .
$$

3. Branching processes in random environments with spatial motion. This section is devoted to the precise construction of branching particle systems with branching affected by a random environment described briefly in the Introduction. At the end of the section we present the main result of this paper, which gives the limiting behavior of these particle systems.

We start with some notation. Let $M_{F}(E)$ be the space of finite measures on $(E, \mathscr{B}(E))$ with the weak topology and $D_{E}[0, \infty)\left(\right.$ resp. $\left.C_{E}[0, \infty)\right)$ be the space of cadlag (resp. continuous) $E$-valued functions on $[0, \infty)$ endowed with the Skorohod topology.

To define the model, let $A$ be the generator of a Feller process in $E$, append to the state space $E$ a cemetery state $\Lambda$ and adopt the convention that $\phi(\Lambda)=0$ for all functions $\phi: E \rightarrow \mathbb{R}$. Let $K_{n}$ be the number of particles alive at time 0 , spatially distributed in $E$ at time 0 at the points $x_{1}, \ldots, x_{K_{n}}$ and defining the initial measure

$$
m_{n}=\sum_{i=1}^{K_{n}} \delta_{x_{i}}, \quad x_{i} \in E, \quad i=1,2, \ldots, K_{n} .
$$


In order to label our particles, define the family of multiindices

$$
I \equiv\left\{\alpha=\left(\alpha_{0}, \alpha_{1}, \ldots, \alpha_{N}\right): \alpha_{0} \in \mathbb{N}, \alpha_{i}=1,2, i \geq 1, N \geq 0\right\} .
$$

Define the "length" of $\alpha$ by $|\alpha|=N$ and set $\left.\alpha\right|_{i}=\left(\alpha_{0}, \ldots, \alpha_{i}\right)$ and $\alpha-i=$ $\left(\alpha_{0}, \ldots, \alpha_{|\alpha|-i}\right)$. We induce a partial order on $I$ by setting

$$
\beta \prec \alpha \Leftrightarrow \beta=\left.\alpha\right|_{i} \quad \text { for some } i \leq|\alpha|,
$$

and for any $t>0$ write $\alpha \sim_{n} t$ if and only if

$$
\frac{|\alpha|}{n} \leq t<\frac{1+|\alpha|}{n} \text {. }
$$

We shall now define the tree $\left\{Y^{\alpha, n}: \alpha_{0} \leq K_{n}\right\}$ of processes. Let $\left\{Y^{\alpha, n}\right.$ : $\left.\alpha_{0} \leq K_{n},|\alpha|=0\right\}$ be a collection of independent Feller processes with generator $A$, stopped at time $t=1 / n$, such that

$$
Y^{\alpha, n}(0)=x_{\alpha_{0}} .
$$

We can now define the tree of processes recursively. For each $k \geq 1$ let $\left\{Y^{\alpha, n}: \alpha_{0} \leq K_{n},|\alpha|=k\right\}$ be a collection of Feller processes with generator $A$, stopped at time $t=(|\alpha|+1) / n$, which are conditionally independent given the $\sigma$-algebra $\sigma\left\{Y^{\alpha, n},|\alpha|<k\right\}$ and for which

$$
Y^{\alpha, n}(t)=Y^{\alpha-1, n}(t), \quad t \leq|\alpha| / n .
$$

Eventually we shall thin out our tree, but in order to do it we need additional random variables, whose distribution depends on the random environment. First we introduce the environment. Let $C_{l}(E)$ be the space of continuous functions on $E$ with limits at $\infty$. Set

$$
\begin{aligned}
\tilde{C}_{l}(E \times E) \equiv\{f \in \bar{C}(E \times E): & f(\cdot, y) \in C_{l}(E) \\
& \left.\forall y \in E ; f(x, \cdot) \in C_{l}(E), \forall x \in E\right\} .
\end{aligned}
$$

Let $\left\{\xi_{k}(\cdot), k \geq 0\right\}$ be independent, identically distributed random fields on $E$ ( $\xi_{k}$ determines the environment) such that

$$
\begin{aligned}
E\left[\left|\xi_{k}(x)\right|^{3}\right] & <\infty, & & \forall x \in E, \forall k, \\
P\left(\xi_{k}(x)>z\right) & =P\left(\xi_{k}(x)<-z\right), & & \forall x \in E, z \in \mathbb{R}, \forall k .
\end{aligned}
$$

Define

$$
\begin{aligned}
\xi_{k}^{(n)}(x) & \equiv \begin{cases}\sqrt{n}, & \xi_{k}(x)>\sqrt{n}, \\
-\sqrt{n}, & \xi_{k}(x)<-\sqrt{n}, \\
\xi_{k}(x), & \text { otherwise, }\end{cases} \\
g_{n}(x, y) & \equiv E\left[\xi_{k}^{(n)}(x) \xi_{k}^{(n)}(y)\right], \\
g(x, y) & \equiv E\left[\xi_{k}(x) \xi_{k}(y)\right] \quad \forall x, y \in E, \forall k,
\end{aligned}
$$

and assume that

$$
g(\cdot, \cdot) \in \tilde{C}_{l}(E \times E) .
$$


For each $n$ let $\left\{N^{\alpha, n}, \alpha \in I\right\}$ be a family of random variables, such that the $\left\{N^{\alpha, n},|\alpha|=k\right\}$ are conditionally independent given $\xi_{k}^{(n)}$ for each $k$, and the conditional probability law of $N^{\alpha, n},|\alpha|=k$ is given by

$$
\begin{aligned}
& P\left(N^{\alpha, n}=2 \mid \xi_{k}, Y^{\alpha, n}\left(\frac{|\alpha|+1}{n}\right)\right)=\frac{1}{2}+\frac{\xi_{k}^{(n)}\left(Y^{\alpha, n}((|\alpha|+1) / n)\right)}{2 \sqrt{n}}, \\
& P\left(N^{\alpha, n}=0 \mid \xi_{k}, Y^{\alpha, n}\left(\frac{|\alpha|+1}{n}\right)\right)=\frac{1}{2}-\frac{\xi_{k}^{(n)}\left(Y^{\alpha, n}((|\alpha|+1) / n)\right)}{2 \sqrt{n}} .
\end{aligned}
$$

Note that our assumptions on $\xi_{k}^{(n)}$ and $N^{\alpha, n}$ are in agreement with those of Kurtz in his case. In particular:

1. The uniform boundedness of the third moment of $X_{k, m}^{(n)}$ corresponds to the relevant condition on $\xi_{k}$.

2. The second moment condition $\lim _{n \rightarrow \infty} n E\left[\left(M_{m}^{(n)}-1\right)^{2}\right]=\gamma$ corresponds to $\lim _{n \rightarrow \infty} g_{n}(x, y)=g(x, y)$ for each $x, y$.

3. The parameter $\beta$ (resp. $\sigma^{2}$ ) corresponds to 0 (resp. 1).

At this point we have to introduce the branching, which we do by thinning the full tree of processes $Y^{\alpha, n}$. For each $\alpha \in I$ define the stopping time

$$
\tau^{\alpha, n}= \begin{cases}0, & \text { if } \alpha_{0}>K_{n}, \\ \min _{0 \leq i \leq|\alpha|}\left\{\frac{i+1}{n}: N^{\left.\alpha\right|_{i}}=0\right\}, & \text { if this set } \neq \varnothing \text { and } \alpha_{0} \leq K_{n}, \\ \frac{1+|\alpha|}{n}, & \text { otherwise. }\end{cases}
$$

We can now finally define the particle paths of interest to us by setting, for each $\alpha \in I$,

$$
X^{\alpha, n}(t)= \begin{cases}Y^{\alpha, n}(t), & \text { if } t<\tau^{\alpha, n} \\ \Lambda, & \text { if } t \geq \tau^{\alpha, n}\end{cases}
$$

The measure-valued process for the finite system of particles is now defined as

$$
X_{t}^{n}(B)=\frac{\#\left\{X^{\alpha, n}(t) \in B: \alpha \sim_{n} t\right\}}{n} .
$$

We shall define the following filtration:

$$
\begin{array}{r}
\mathscr{F}_{t}^{n} \equiv \sigma\left(Y^{\alpha, n}, N^{\alpha, n}:|\alpha|<j\right) \vee\left[\bigcap_{s>t} \sigma\left(Y^{\alpha, n}(s):|\alpha|=j+1\right)\right] \\
\text { if } \frac{j}{n} \leq t<\frac{j+1}{n}, \forall j \geq 0 .
\end{array}
$$

Let $\mathscr{D}(A)$ be the domain of $A$. Here is the main result of the paper. 
Theorem 3.1. Let $g \in \tilde{C}_{l}(E \times E)$. Assume that $X_{0}^{n} \Rightarrow m$ in $M_{F}(E)$. Then $X^{n} \Rightarrow X$, where $X \in C_{M_{F}(E)}[0, \infty)$ is the unique solution of the following martingale problem:

$$
\begin{aligned}
& \left\{\begin{array}{l}
\text { For all } \phi \in \mathscr{D}(A), \\
\qquad Z_{t}(\phi)=X_{t}(\phi)-m(\phi)-\int_{0}^{t} X_{s}(A \phi) d s
\end{array}\right. \\
& \left\{\begin{array}{l}
\text { is an } \mathscr{F}_{t}^{X} \text { continuous square-integrable martingale } \\
\text { such that } Z_{0}(\phi)=0 \text { and } \\
\qquad\langle Z(\phi)\rangle_{t}=\int_{0}^{t} X_{s}\left(\phi^{2}\right) d s
\end{array}\right. \\
& +\int_{0}^{t} \int_{E \times E} g(x, y) \phi(x) \phi(y) X_{s}(d x) X_{s}(d y) d s .
\end{aligned}
$$

Modulo the following small subsection, the remainder of this paper is devoted to the proof of Theorem 3.1.

Some comments on the limit process. It is notable that although in (3.5) $Z$ is an $L^{2}$-martingale measure in the sense of Walsh's definition [21], it is not orthogonal, in contrast to the case of the usual superprocess. This is one of the aspects of this model that makes it both interesting and challenging. In particular, establishing uniqueness for the solution of (3.5), which is both of intrinsic interest and necessary for proving the weak convergence of the theorem, becomes a particularly challenging task.

In this paper we do not consider the properties of the limit process, since many of them seem to be similar to already studied models. For example, it is not hard to check that the Hausdorff dimension of the support of $X$ in the case $E=\mathbb{R}^{d}, A=-(-\Delta)^{\alpha / 2}$ and $g=$ constant is the same as for the corresponding standard superprocess. (We also believe that for any sufficiently regular $g$ the situation is the same.)

Similarly, under the same assumptions but with $X_{0}=\lambda=$ Lebesgue measure (our model can be easily generalized to the case of infinite initial measures), it follows that for small dimensions $(d \leq \alpha), X_{t} \Rightarrow 0$ as $t \rightarrow \infty$, as does the corresponding standard superprocess. For $d>\alpha$ the limiting behavior depends on $g$ much as in the case of linear stochastic partial differential equations (for $\alpha=2$, see, e.g., [2]).

An interesting extension to our setup arises in the case that $g$ is not a regular function, but, for example, is of the form $g(x, y)=\delta_{0}(x-y)$, where $\delta_{0}$ is the Dirac delta function. In the case $A=\frac{1}{2} \Delta$ and $d=1$ this can be treated as a particular case of a model considered by Mueller and Perkins [16]. They proved the existence of the weak solution of the following stochastic partial differential equation:

$$
u_{t}=\frac{1}{2} u_{x x}+a(u) \dot{W},
$$


where $a(\cdot)$ is a function satisfying some growth condition, $\dot{W}=\dot{W}(t, x)$ and is a two-parameter white noise. Taking $a(u)=\sqrt{u(1+u)}$ and defining $X_{t}(d x)$ $=u(t, x) d x$, one can check that such $X$ solves the martingale problem (3.5) with $g(x, y)=\delta_{0}(x-y)$ and so we recover a "special case" of our model. In [16] it is noted that uniqueness in law of the solution of (3.6) is not known for the general case. However, in our situation, in which $a(u)=\sqrt{u(1+u)}$, the uniqueness is easily verified by adapting the arguments developed in Section 4.2. The question of uniqueness for (3.6) in the general case seems to be very interesting, but considerably harder, and probably will be the subject of another paper.

4. Proofs. The proof of weak convergence involves two steps:

1. Tightness.

2. Identification of the limit; in our case this is equivalent to the proof of uniqueness of the solution for the martingale problem (3.5).

4.1. Tightness. In this subsection we shall prove the following result.

LEMMA 4.1. The sequence $\left\{X^{n}\right\}$ is tight in $D_{M_{F}(E)}[0, \infty)$, and each limit process is continuous and satisfies the martingale problem (3.5).

We shall not go into the details of the proof, which are similar to those in [5], Chapter 7, where the more complicated case of historical processes is treated.

One of the basic tools used in establishing tightness for $M_{F}(E)$-valued processes involves reducing this problem to the tightness of real-valued processes. According to the following theorem (which is an easy consequence of Theorem 3.7.1 and Definition 3.2.1 of [3]), if $E$ is compact, then in order to show that the sequence $\left\{X^{(n)}\right\}$ of $M_{F}(E)$-valued processes is tight one merely needs to verify that, for each "good" function $\phi$, the sequence of real-valued processes $\left\{X^{(n)}(\phi)\right\}$ is tight.

Theorem 4.2. Assume that $E$ is a compact Polish space. Let $S=\left\{\phi_{k}\right.$ : $k \geq 0\}$ be a dense subset in $C(E)$ with $\phi_{0}=1$. Let $\left\{X^{(n)}\right\}$ be a sequence of processes with values in $M_{F}(E)$. Then $\left\{X^{(n)}\right\}$ is tight in $D_{M_{F}(E)}[0, \infty)$ and all limit points are in $C_{M_{F}(E)}[0, \infty)$ if and only if, for each $\phi \in S,\left\{X^{(n)}(\phi)\right\}$ is tight and all limit points are in $C_{\mathbb{R}}[0, \infty)\left[\right.$ i.e., $\left\{X^{(n)}(\phi)\right\}$ is $C$-tight $]$.

Before we can continue, we require some further notation:

$$
\begin{aligned}
& a_{n} \equiv 1 / n \quad \forall n>0, \\
& s_{n} \equiv[s n] / n \quad \forall s \geq 0, n>0 .
\end{aligned}
$$


Let $\alpha \sim s, s \in\left[i_{n}, i_{n}+a_{n}\right)$. Fix $\phi \in \mathscr{D}(A)$. Furthermore define

$$
M_{s}^{\alpha, i_{n}}(\phi)=\left\{\begin{array}{cc}
\phi\left(Y^{\alpha, n}(s)\right)-\phi\left(Y^{\alpha, n}\left(i_{n}\right)\right) & \\
-\int_{i_{n}}^{s} A \phi\left(Y^{\alpha, n}(r)\right) d r, & \text { if } X^{\alpha, n}\left(i_{n}\right) \neq \Lambda, \\
0, & \text { if } X^{\alpha, n}\left(i_{n}\right)=\Lambda .
\end{array}\right.
$$

Clearly, $\left\{\left(M_{s}^{\alpha, i_{n}}(\phi), \mathscr{F}_{s}^{n}\right), s \in\left[i_{n}, i_{n}+a_{n}\right)\right\}$ is a martingale for each $i \geq 0$.

It is easy to check that

$$
X_{t}^{n}(\phi)=X_{0}^{n}(\phi)+N_{t}^{(n)}(\phi)+M_{t}^{(n)}(\phi)+Z_{t}^{(n)}(\phi)+\int_{0}^{t} X_{s}^{n}(A \phi) d s,
$$

where

$$
\begin{aligned}
M_{t}^{(n)}(\phi) & =n^{-1} \sum_{s_{n}<t_{n}} \sum_{\alpha \sim_{n} s_{n}} M_{s_{n}+\alpha_{n}}^{\alpha, s_{n}}(\phi) N^{\alpha, n}+n^{-1} \sum_{\alpha \sim t_{n}} M_{t}^{\alpha, t_{n}}(\phi), \\
N_{t}^{(n)}(\phi) & =n^{-1} \sum_{s_{n}<t_{n}} \sum_{\alpha \sim_{n} s_{n}} \int_{s_{n}}^{s_{n}+a_{n}} A \phi\left(X^{\alpha, n}(r)\right) d r\left(N^{\alpha, n}-1\right), \\
Z_{t}^{(n)}(\phi) & =n^{-1} \sum_{s_{n}<t_{n}} \sum_{\alpha \sim_{n} s_{n}} \phi\left(X^{\alpha, n}\left(s_{n}\right)\right)\left(N^{\alpha, n}-1\right) .
\end{aligned}
$$

It can be easily verified that $M^{(n)}, N^{(n)}, Z^{(n)}$ are $\mathscr{F}^{n}$-martingales, such that $M^{(n)}, N^{(n)}$ are closely related to the motion of the particles and $Z^{(n)}$ is related to the branching. Let $Y, \tilde{Y}$ be two independent Feller processes, each with generator $A$, and let $T(t)$ be the semigroup corresponding to the two-dimensional process $(Y, \tilde{Y})$. Then simple calculations show that

$$
\begin{aligned}
\left\langle Z^{(n)}(\phi)\right\rangle_{t}= & \sum_{s_{n}<t_{n}} X_{s_{n}}^{n}\left(\phi^{2}\right) a_{n} \\
& +\sum_{s_{n}<t_{n}} \int_{E \times E} \phi(x) \phi(y) T\left(a_{n}\right) g_{n}(x, y) X_{s_{n}}^{n}(d x) X_{s_{n}}^{n}(d y) a_{n} \\
& -n^{-1} \sum_{s_{n}<t_{n}} \int_{E} \phi(x) T\left(a_{n}\right) g_{n}(x, x) X_{s_{n}}^{n}(d x) a_{n} \\
= & \int_{0}^{t_{n}} X_{s_{n}}^{n}\left(\phi^{2}\right) d s \\
& +\int_{0}^{t_{n}} \int_{E \times E} \phi(x) \phi(y) T\left(a_{n}\right) g_{n}(x, y) X_{s_{n}}^{n}(d x) X_{s_{n}}^{n}(d y) d s \\
& -n^{-1} \int_{0}^{t_{n}} \int_{E} X_{s_{n}}^{n}\left(\phi T\left(a_{n}\right) g_{n}(\cdot, \cdot)\right) d s .
\end{aligned}
$$

From the properties of the generators of Feller semigroups we have that $N^{(n)}(1) \equiv 0, M^{(n)}(1) \equiv 0$. Hence, an application of Gronwall's and Doob's inequalities yields the following result. 
LEMMA 4.3. For each $T>0, E\left[\sup _{t \leq T} X_{t}^{n}(1)^{2}\right]$ is bounded uniformly in $n$.

It is much easier first to prove Theorem 4.1 for $E$ compact. The main steps (without proof) that lead to a proof of tightness in the case of compact $E$ are as follows:

(i) Using the number boundedness of $E\left[\sup _{t \leq T} X_{t}^{n}(1)^{2}\right]$ and the Feller property for the random motion, we can prove the following result.

LEMMA 4.4. For each $T>0, \phi \in \mathscr{D}(A)$,

$$
\begin{aligned}
& \lim _{n \rightarrow \infty} E\left[\sup _{s \leq T} M_{s}^{(n)}(\phi)^{2}\right]=0, \\
& \lim _{n \rightarrow \infty} E\left[\sup _{s \leq T} N_{s}^{(n)}(\phi)^{2}\right]=0 .
\end{aligned}
$$

(ii) The following step includes establishing tightness for $\left\{Z^{(n)}(\phi)\right\}$ and $\left\{\left\langle Z^{(n)}(\phi)\right\rangle\right\}$ and some additional moment properties. Since $g(\cdot, \cdot)$ and $\phi(\cdot)$ are bounded functions, it follows that the crucial arguments leading to the proof of tightness for a sequence of branching processes in a random environment (without spatial motion) do not change. Hence, following a similar procedure to that in the proof of Theorem 8.1 of [10] (see also Proposition 6.3.26 of [12]), one can obtain the following result.

Lemma 4.5. (a) The sequence $\left\{Z^{(n)}(\phi)\right\},\left\{\left\langle Z^{(n)}(\phi)\right\rangle\right\}$ are $C$-tight sequences of processes in $D_{\mathbb{R}}[0, \infty)$.

(b) The qualities $\left\{\sup _{t \leq T} X_{t}^{n}(\phi)^{2}\right\},\left\{\sup _{t \leq T} Z_{t}^{(n)}(\phi)^{2}\right\}$ are uniformly integrable random variables for each $T \geq 0$.

(iii) Define $C_{t}^{(n)}(\phi) \equiv \int_{0}^{t} X_{s}^{n}(A \phi) d s$. Then

$$
\begin{aligned}
\left|C_{t}^{(n)}(\phi)-C_{s}^{(n)}(\phi)\right| & \leq|t-s| \sup _{r \leq t} X_{r}^{n}(|A \phi|) \\
& \leq\|A \phi\| \sup _{r \leq t} X_{r}^{n}(1) .
\end{aligned}
$$

Checking the conditions of Proposition 6.3.26 of [12] yields the following result.

Lemma 4.6. The sequence $\left\{C^{(n)}(\phi)\right\}$ is $C$-tight in $D_{\mathbb{R}}[0, \infty)$.

(iv) The sequences $\left\{C^{(n)}(\phi)\right\}$ and $\left\{Z^{(n)}(\phi)\right\}$ are $C$-tight; thus, by Corollary 6.3.33 of [12], $\left\{C^{(n)}(\phi)+Z^{(n)}(\phi)\right\}$ is $C$-tight. By (4.1) and Lemma 4.4 we obtain that $\left\{X^{n}(\phi)\right\}$ is $C$-tight.

LeMma 4.7. The sequence $\left\{X^{n}\right\}$ is tight in $D_{M_{F}(E)}[0, \infty)$ and all limit points are in $C_{M_{F}(E)}[0, \infty)$. 
Proof. The family $\mathscr{D}(A)$ is dense in $C(E)$ and the conclusion follows immediately from Theorem 4.2 .

(v) Assume that $X$ is one of the limit points of $\left\{X^{n}\right\}$. Using Lemma 4.5(b) and martingale techniques, we can derive that

$$
X_{t}(\phi)=m(\phi)+Z_{t}(\phi)+\int_{0}^{t} X_{s}(A \phi) d s,
$$

where $Z_{t}(\phi)$ is a square-integrable $\mathscr{F}_{t}^{X}$-martingale and

$$
\langle Z(\phi)\rangle_{t}=\int_{0}^{t} X_{s}\left(\phi^{2}\right) d s+\int_{0}^{t} \int_{E \times E} g(x, y) \phi(x) \phi(y) X_{s}(d x) X_{s}(d y) d s .
$$

The last step completes the proof of Theorem 4.1 for compact $E$.

In the case of locally compact $E$, we write $\dot{E} \equiv E \cup\{\infty\}$ for the one-point compactification of $E$. As above, we get the tightness of $X^{n}$ in $D_{M_{F}(\dot{E})}[0, \infty)$. Let $X$ be a limit point of $X^{n}$ in $D_{M_{F}(\dot{E})}[0, \infty)$. Choosing $\left\{f_{n}\right\} \in \mathscr{D}(A)$ such that $f_{n} \rightarrow 1_{\infty}, A f_{n} \rightarrow 0$ boundedly pointwise, it can be easily verified that

$$
\lim _{n \rightarrow \infty} E\left[\sup _{t \leq T} X_{t}\left(f_{n}\right)^{2}\right]=0 \quad \forall T>0 .
$$

Thus, by dominated convergence, $E\left[\sup _{t \leq T} X_{t}(\infty)^{2}\right]=0$ and so

$$
X_{t}(\{\infty\})=0 \quad \forall t \text {, a.s., }
$$

from which it follows that $\left\{X^{n}\right\}$ is tight in $D_{M_{F}(E)}[0, \infty)$.

4.2. Uniqueness. In this subsection we shall prove the hard part of Theorem 3.1, that the martingale problem (3.5) has a unique solution. Our proof is based on Theorem 1.3, so that we need to construct some approximating sequence of processes. We shall start with some auxiliary results.

Note first that we shall use the convention that $S_{t}=0$ for $t<0$, where $\left\{S_{t}\right\}$ is the semigroup generated by $A$, and abbreviate "bounded pointwise" by bp.

As a first step, note that via Itô's formula one can check that every solution of (3.5) is also a solution of the martingale problem for $\left(A_{1}, \delta_{m}\right)$, where

$$
\begin{aligned}
& A_{1} \equiv\{ f(\mu(\phi)), f^{\prime}(\mu(\phi)) \mu(A \phi) \\
&+\frac{1}{2} f^{\prime \prime}(\mu(\phi))\left(\mu\left(\phi^{2}\right)+\int_{E \times E} g(x, y) \phi(x) \phi(y) \mu(d x) \mu(d y)\right): \\
&\left.f \in \bar{C}^{2}\left(\mathbb{R}_{+}\right), \phi \in \mathscr{D}(A)_{++}\right\} .
\end{aligned}
$$

Hence, in order to prove uniqueness for (3.5), it is sufficient to prove uniqueness for $\left(A_{1}, \delta_{m}\right)$. In fact, the following result shows that the two martingale problems are equivalent. 
TheOREM 4.8. Let $X$ be an $M_{F}(E)$-valued process with $P X_{0}^{-1}=\nu \in$ $\mathscr{P}\left(M_{F}(E)\right)$. It is a solution of the martingale problem for $\left(A_{1}, \nu\right)$ if and only if

for all $\phi \in \mathscr{D}(A)$,

$$
Z_{t}(\phi)=X_{t}(\phi)-X_{0}(\phi)-\int_{0}^{t} X_{s}(A \phi) d s
$$

is an $\mathscr{F}_{t}^{X}$-continuous locally square-integrable martingale

such that $Z_{0}(\phi)=0$ and

$$
\begin{aligned}
\langle Z(\phi)\rangle_{t}= & \int_{0}^{t} X_{s}\left(\phi^{2}\right) d s \\
& +\int_{0}^{t} \int_{E \times E} g(x, y) \phi(x) \phi(y) X_{s}(d x) X_{s}(d y) d s
\end{aligned}
$$

REMARK 4.9. From the above theorem it immediately follows that any solution of the martingale problem for $\left(A_{1}, \nu\right)$ is continuous.

REMARK 4.10. Note that (4.4) differs from (3.5) in that $Z(\phi)$ can be a local martingale.

Proof. (i) Applying Itô's formula, we get that if $X$ satisfies (4.4), then it is a solution of the martingale problem for $\left(A_{1}, \nu\right)$ (we shall also need the fact that each bounded local martingale is in fact martingale).

(ii) Let $X$ be a solution of the martingale problem for $\left(A_{1}, \nu\right)$. Then, for each $\phi \in \mathscr{D}(\mathrm{A})_{++}$,

$$
\begin{aligned}
\exp ( & \left.-\varepsilon X_{t}(\phi)\right) X_{t}(\phi)-\int_{0}^{t} \exp \left(-\varepsilon X_{s}(\phi)\right) \\
\times & \left\{\left(-\varepsilon X_{s}(\phi)+1\right) X_{s}(A \phi)+\frac{1}{2}\left(\varepsilon^{2} X_{s}(\phi)-2 \varepsilon\right)\right. \\
& \left.\times\left(X_{s}\left(\phi^{2}\right)+\int_{E \times E} g(x, y) \phi(x) \phi(y) X_{s}(d x) X_{s}(d y)\right)\right\} d s
\end{aligned}
$$

is an $\mathscr{F}_{t}^{X}$-martingale. Letting $\varepsilon \downarrow 0$, we easily get by stopping time arguments that

$$
Z_{t}(\phi)=X_{t}(\phi)-X_{0}(\phi)-\int_{0}^{t} X_{s}(A \phi) d s
$$

is a local martingale for all $\phi \in \mathscr{D}(A)_{++}$. Since an arbitrary $\phi \in \mathscr{D}(A)$ is bounded by some constant $k\left[\sup _{x \in E}|\phi(x)|<k\right]$, we get that $\phi+k, k \in$ $\mathscr{D}(A)_{++}$and, hence,

$$
Z_{t}(\phi)=Z_{t}(\phi+k)-Z_{t}(k)
$$


is a local martingale. Furthermore,

$$
\begin{aligned}
\tilde{M}_{t} \equiv \exp \left(-X_{t}(\phi)\right)-\int_{0}^{t} \exp ( & \left.X_{s}(\phi)\right)\left(X_{s}\left(-A \phi+\frac{1}{2} \phi^{2}\right)\right. \\
& \left.+\frac{1}{2} \int_{E \times E} g(x, y) \phi(x) \phi(y) X_{s}(d x) X_{s}(d y)\right) d s
\end{aligned}
$$

is an $\mathscr{F}_{t}^{X}$-martingale for each $\phi \in \mathscr{D}(A)_{++}$. Now, applying Itô's formula to the semimartingale $V_{t} \equiv \exp \left\{-X_{t}(\phi)\right\}$ and the continuous process

$$
\begin{aligned}
\tilde{V}_{t} \equiv \exp \left\{\int_{0}^{t}[\right. & X_{s}\left(A \phi-\frac{1}{2} \phi^{2}\right) \\
& \left.\left.-\frac{1}{2} \int_{E \times E} g(x, y) \phi(x) \phi(y) X_{s}(d x) X_{s}(d y)\right] d s\right\},
\end{aligned}
$$

we get that

$$
\begin{gathered}
V_{t} \tilde{V}_{t}=V_{0} \tilde{V}_{0}+\int_{0}^{t} V_{s-} d \tilde{V}_{s}+\int_{0}^{t} \tilde{V}_{s} d V_{s} \\
=V_{0} \tilde{V}_{0}+\int_{0}^{t} \exp \left\{\int _ { 0 } ^ { s } \left[X_{u}\left(A \phi-\frac{1}{2} \phi^{2}\right)\right.\right. \\
\left.\quad-\frac{1}{2} \int_{E \times E} g(x, y) \phi(x) \phi(y) X_{u}(d x) X_{u}(d y) d u\right\} d \tilde{M}_{s} \\
=\exp \left\{-X_{t}(\phi)+\int_{0}^{t} X_{s}\left(A \phi-\frac{1}{2} \phi^{2}\right)\right. \\
\left.\left.\quad-\frac{1}{2} \int_{E \times E} g(x, y) \phi(x) \phi(y) X_{s}(d x) X_{s}(d y)\right] d s\right\} .
\end{gathered}
$$

is a local martingale. Now the continuity and quadratic variation of $Z_{t}(\phi)$ follow in much the same way as in (ii) $\rightarrow$ (iii) of Theorem 1.3 of [19].

Let $S_{t}$ be the semigroup generated by $A$ and let $X$ be a solution of the martingale problem for $\left(A_{1}, \nu\right)$. It is easy to verify that

$M_{t}^{T}(\phi) \equiv \exp \left(-X_{t}\left(S_{T-t} \phi\right)\right)$

$$
\begin{aligned}
-\int_{0}^{t} \exp \left(-X_{s}\left(S_{T-s} \phi\right)\right)( & X_{s}\left(\frac{1}{2} S_{T-s}(\phi)^{2}\right) \\
& +\frac{1}{2} \int_{E \times E} g(x, y)\left(S_{T-s} \phi\right)(x)\left(S_{T-s} \phi\right)(y) \\
& \left.\times X_{s}(d x) X_{s}(d y)\right) d s
\end{aligned}
$$

is an $\mathscr{F}_{t}^{X}$-martingale on $0 \leq t \leq T$ for each $T>0$ and $\phi \in B(E)_{++}$. This result may be obtained, for example, by applying Lemma 4.3 .4 of [7] for 
$\phi \in \mathscr{D}(A)_{++}$and then going to the limit [using the fact that $\mathscr{D}(A)_{++}$is dense in $C_{l}(E)_{+}$and $C_{l}(E)_{+}$is bp-dense in $\left.B(E)_{+}\right]$.

We want to state now one auxiliary lemma about the existence of the $k$ th moment of $X$.

Lemma 4.11. Suppose $k \geq 2$. Let $X$ be the solution of the martingale problem for $\left(A_{1}, \nu\right)$, where $\nu$ has the kth moment, in the sense that $E_{\nu}\left[X_{0}(1)^{k}\right]$ $<\infty$. Then $E\left[X_{t}(1)^{k}\right]<\infty$ for each $t>0$.

Proof. The proof is standard and uses Gronwall's inequality and stopping time arguments. Let $\tau_{n}=\inf \left\{t \geq 0: X_{t}(1) \geq n\right\}$. Then, by Itô's formula,

$$
\begin{aligned}
X_{t \wedge \tau_{n}}(1)^{k}= & X_{0}(1)^{k}+k(k-1) \int_{0}^{t} X_{s \wedge \tau_{n}}(1)^{k-2} d\langle Z(\phi)\rangle_{s \wedge \tau_{n}} \\
& + \text { local martingale. }
\end{aligned}
$$

Since all the terms are bounded, we get that the local martingale is actually a martingale and, hence,

$$
\begin{aligned}
X_{t \wedge \tau_{n}}(1)^{k} \leq X_{0}(1)^{k}+k(k-1) & \int_{0}^{t} X_{s \wedge \tau_{n}}(1)^{k-2} \\
& \times\left(X_{s \wedge \tau_{n}}(1)+\|g\| X_{s \wedge \tau_{n}}(1)^{2}\right) d s+\text { martingale, }
\end{aligned}
$$

where $\|\cdot\|$ denotes the supremum norm. Furthermore,

$$
\begin{aligned}
E\left[X_{t \wedge \tau_{n}}(1)^{k}\right] \leq & E\left[X_{0}(1)^{k}\right] \\
& +k(k-1) \int_{0}^{t}\left[E\left[X_{s \wedge \tau_{n}}(1)^{k-1}\right]+\|g\| E\left[X_{s \wedge \tau_{n}}(1)^{k}\right]\right] d s \\
\leq & E\left[X_{0}(1)^{k}\right]+k(k-1) \int_{0}^{t}\left[1+(1+\|g\|) E\left[X_{s \wedge \tau_{n}}(1)^{k}\right]\right] d s .
\end{aligned}
$$

Thus, by Gronwall's inequality,

$$
E\left[X_{t \wedge \tau_{n}}(1)^{k}\right] \leq C_{t} e^{\|g\| t},
$$

where the constant $C_{t}$ is independent of $n$. Letting $n \rightarrow \infty$ and applying the monotone convergence theorem, we are done.

CoRollary 4.12. If $\nu$ has a second moment, then $M_{t}^{T}(\phi)$ in (4.5) is an $\mathscr{F}_{t}{ }^{X}$-martingale on $0 \leq t \leq T$ for each $\phi \in B(E)_{+}$.

In order to apply Theorem 1.3 we shall construct a sequence of approximating dual processes. The motivation behind our construction is as follows. 
Suppose that for each $\phi \in C_{l}(E)_{+}$there exists a $C_{l}(E)_{+}$-valued process $Y$ which solves the following martingale problem:

$$
\begin{aligned}
& \text { for all } \mu \in M_{F}(E) \text { and } T>0 \text {, } \\
& \qquad M_{t}^{T}(\mu)=\mu\left(S_{T-t} Y_{t}\right)-\mu\left(S_{T} \phi\right)-\int_{0}^{t} \mu\left(S_{T-s}\left(\frac{1}{2} Y_{s}^{2}\right)\right) d s,
\end{aligned}
$$

is an $\mathscr{F}_{t}^{Y}$-continuous square-integrable martingale on

$0 \leq t \leq T$ such that $M_{0}^{T}(\mu)=0$ and

$$
\begin{array}{rl}
\left\langle M^{T}(\mu)\right\rangle_{t}=\int_{0}^{t} \int_{E \times E} & g(x, y) Y_{s}(x) Y_{s}(y) \\
& \times S_{T-s}^{*} \mu(d x) S_{T-s}^{*} \mu(d y) d s, \quad t \leq T .
\end{array}
$$

Then, applying arguments of Chapter 4.4 of [7], we can show that if $X$ is any solution of the martingale problem $\left(A_{1}, \nu\right)$, independent of $Y$, then

$$
E\left[\exp \left(-X_{t}(\phi)\right)\right]=E\left[\exp \left(-X_{0}\left(Y_{t}\right)\right)\right], \quad \forall t \geq 0
$$

and (once again by arguments of Chapter 4.4 of [7]) this gives us uniqueness for the original martingale problem $\left(A_{1}, \nu\right)$. The only problem is the existence of the process $Y$. For a special case $g=$ constant, the existence of $Y$ can be easily verified. For a more general $g$, the situation is rather more complicated. Thus, we intend to construct a sequence of processes that satisfies the conditions of Theorem 1.3.

Let $g(\cdot, \cdot)$ be an arbitrary nonnegative-definite function in $\tilde{C}_{l}(E \times E)$ and let $\tilde{\xi}$ be a measurable version of a Gaussian mean-zero random field on $E$ with covariance function $g$ (the existence of such a modification follows, for example, from Theorem 9.4.2 of [20]). Define the following sequence of bounded random functions:

$$
\xi^{(n)}(x)= \begin{cases}\sqrt{n}, & \tilde{\xi}(x)>\sqrt{n} \\ -\sqrt{n}, & \tilde{\xi}(x)<-\sqrt{n} \\ \tilde{\xi}(x), & \text { otherwise }\end{cases}
$$

for each $x \in E$. Define

$$
\begin{aligned}
g_{n}(x, y) & \equiv E\left[\xi^{(n)}(x) \xi^{(n)}(y)\right], \\
\tilde{h}_{n}(x, y, z) & \equiv E\left[\xi^{(n)}(x) \xi^{(n)}(y) \xi^{(n)}(z)\right] .
\end{aligned}
$$

Let $\left\{\tau_{i}^{(n)}, i=1,2, \ldots\right\}$ be independent exponential random variables with parameter $n$ and let $\left\{\xi_{i}^{(n)}, i=1,2, \ldots\right\}$ be independent copies of $\xi^{(n)}$, such that $\left\{\tau_{i}^{(n)}\right\}$ and $\left\{\xi_{i}^{(n)}\right\}$ are mutually independent. Define also the following random variables:

$$
T_{k}^{(n)}=\sum_{i=1}^{k} \tau_{i}^{(n)}, \quad k_{1} n \geq 1 .
$$


Now for each $n$ we construct the process $Y^{(n)}$, with sample paths in $D_{B(E)_{+}}[0, \infty)$ [to be more precise, we can endow $B(E)$ with the supremum norm and hence define the topology on $B(E)_{+}$as the relative topology], as iterative solutions of the following evolution equations (the existence and uniqueness of solutions of these equations follow, for example, from Theorem 1.1 of $[6])$ :

$Y_{n}^{t}= \begin{cases}Y_{0}^{(n)}=\phi \in C_{l}(E)_{+}, & 0 \leq t<\tau_{1}^{(n)}, \\ S_{t}(\phi)-\int_{0}^{t} S_{t-s}\left(\frac{1}{2} Y_{s}^{(n)^{2}}\right) d s, & \\ S_{t-\tau_{1}^{(n)}}\left(Y_{\tau_{1}^{(n)}-}^{(n)}+\frac{\xi_{1}^{(n)}(\cdot)}{\sqrt{n}} Y_{\tau_{1}^{(n)}-}^{(n)}\right)-\int_{\tau_{1}^{(n)}}^{t} S_{t-s}\left(\frac{1}{2} Y_{s}^{(n)^{2}}\right) d s & \\ S_{t}(\phi)-\int_{0}^{t} S_{t-s}\left(\frac{1}{2} Y_{s}^{(n)^{2}}\right) d s & \\ \quad+S_{t-\tau_{1}^{(n)}}\left(\frac{\xi_{1}^{(n)}(\cdot)}{\sqrt{n}} Y_{\tau_{1}^{(n)}-}^{(n)}\right), & \\ \quad \cdots & \\ S_{t}(\phi)-\int_{0}^{t} S_{t-s}\left(\frac{1}{2} Y_{s}^{(n)^{2}}\right) d s & \\ \quad+\sum_{i=1}^{k} S_{t-T_{i}^{(n)}}\left(\frac{\xi_{i}^{(n)}(\cdot)}{\sqrt{n}} Y_{T_{i}^{(n)}-}^{(n)}\right), & \tau_{1}^{(n)} \leq t<\tau_{2}^{(n)}, \\ \quad \cdots . & \end{cases}$

Since $\left\{\tau_{i}^{(n)}\right\}$ and $\left\{\xi_{i}^{(n)}\right\}$ are mutually independent, we can define the Poisson point process $([11], 1.9)$ :

$$
p^{(n)}: D_{p^{(n)}} \subset(0, \infty) \mapsto B(E),
$$

with countable domain $D_{p^{(n)}}$ defined by

$$
D_{p^{(n)}} \equiv\left\{\tau_{1}^{(n)}, \tau_{1}^{(n)}+\tau_{2}^{(n)}, \ldots, \sum_{i=1}^{k} \tau_{i}^{(n)}, \ldots\right\}
$$

and

$$
p^{(n)}\left(\sum_{i=1}^{k} \tau_{i}^{(n)}\right)=\xi_{k}^{(n)} \quad \forall k \geq 1 .
$$

The corresponding Poisson counting measure is defined as

$$
\begin{aligned}
N^{(n)}(t, U) & =N^{(n)}((0, t] \times U) \\
& \equiv \#\left\{s \in D_{p^{(n)}} ; s \leq t, p^{(n)}(s) \in U\right\} \quad \forall t>0, \forall U \in \mathscr{B}(B(E))
\end{aligned}
$$


and its compensator is

$$
\hat{N}^{(n)}(t, U)=E\left[N^{(n)}(t, U)\right]=n t E_{\xi^{(n)}}\left[1_{U}\right],
$$

where $E_{\xi^{(n)}}$ denotes expectation with respect to the distribution of $\xi^{(n)}$.

Let $\mathscr{F}_{t}^{n} \equiv \bigcap_{\varepsilon>0} \sigma\left\{N^{(n)}(s, U) ; s \leq t+\varepsilon, U \in \mathscr{B}(B(E))\right\}$ and define the spaces

$$
\begin{aligned}
L_{k}^{(n)} \equiv\{f(t, h, w) ; & f \text { is } \mathscr{F}_{t}^{n} \text {-predictable and } \\
& \left.\forall t>0 E\left[\int_{0}^{t} \int_{B(E)}|f(s, h, \cdot)|^{k} \hat{N}^{(n)}(d s, d h)\right]<\infty\right\} .
\end{aligned}
$$

Defining $\tau^{(n)}(s) \equiv \max \left\{\tau \in D_{p^{(n)}} ; \tau \leq s\right\}$ and $N_{s}^{(n)} \equiv N^{(n)}(s, B(E))$, we get from the construction of $Y^{(n)}$ that

$$
\begin{aligned}
\left\|Y_{s}^{(n)}\right\|^{k} & \leq\left\|Y_{\tau^{(n)}(s)}^{(n)}\right\|^{k} \\
& =\left\|Y_{\tau^{(n)}(s)-}^{(n)}\left(1+\frac{\xi_{\tau^{(n)}(s)}^{(n)}}{\sqrt{n}}\right)\right\|^{k} \\
& \leq 2^{k}\left\|Y_{\tau^{(n)}(s)-}^{(n)}\right\|^{k} \leq \cdots \leq\|\phi\|^{k} 2^{k N_{2}^{(n)}}
\end{aligned}
$$

for each $s \geq 0$. The expectation of the last expression is finite for each $k \geq 1$; hence, it is easy to check that, for each $\mu \in M_{F}(E)$ and $T>0$,

$$
f(t, h, \cdot) \equiv \mu\left(S_{T-t}\left(\frac{h}{\sqrt{n}} Y_{t-}^{(n)}\right)\right) \in L_{k}^{(n)} \quad \forall k \geq 1,
$$

and $Y^{(n)}$ is the unique solution of the following stochastic evolution equation:

$$
\begin{aligned}
Y_{t}^{(n)}(x)= & S_{t}(\phi)(x)-\int_{0}^{t} S_{t-s}\left(\frac{1}{2} Y_{s}^{(n)^{2}}\right)(x) d s \\
& +\int_{0}^{t+} \int_{B(E)} S_{t-s}\left(\frac{h}{\sqrt{n}} Y_{s-}^{(n)}\right)(x) N^{(n)}(d s, d h)
\end{aligned}
$$

for all $x \in E$, or

$$
\begin{aligned}
\mu\left(S_{T-t}\left(Y_{t}^{(n)}\right)\right)= & \mu\left(S_{T}(\phi)\right)-\int_{0}^{t} \mu\left(S_{T-s}\left(\frac{1}{2} Y_{s}^{(n)^{2}}\right)\right) d s \\
& +\int_{0}^{t+} \int_{B(E)} \mu\left(S_{T-s}\left(\frac{h}{\sqrt{n}} Y_{s-}^{(n)}\right)\right) N^{(n)}(d s, d h)
\end{aligned}
$$

for each $\mu \in M_{F}(E)$ and $T>0$. From (4.9) and Definition 2.4.1 of [11], it follows that the last expression is a semimartingale; hence, Itô's formula (Theorem 2.5.1 of [11]) immediately yields the following result. 
LEMMA 4.13. For each $\mu \in M_{F}(E), T>0$ and $F \in C^{1}(\mathbb{R})$,

$$
\begin{array}{r}
F\left(\mu\left(S_{T-t} Y_{t}^{(n)}\right)\right)= \\
F\left(\mu\left(S_{T}(\phi)\right)\right)-\int_{0}^{t} F^{\prime}\left(\mu\left(S_{T-s}\left(\frac{1}{2} Y_{s}^{(n)^{2}}\right)\right)\right) d s \\
+\int_{0}^{t+} \int_{B(E)}\left\{F\left(\mu\left(S_{T-s}\left(Y_{s-}^{(n)}+\frac{h}{\sqrt{n}} Y_{s-}^{(n)}\right)\right)\right)\right. \\
\left.-F\left(\mu\left(S_{T-s} Y_{s-}^{(n)}\right)\right)\right\} N^{(n)}(d s, d h)
\end{array}
$$

for $0 \leq t \leq T$.

Corollary 4.14. Let

(i) $F \in \bar{C}^{1}(\mathbb{R})$

or

(ii) $F(x)=x^{k}, x \in \mathbb{R}$ for some $k \geq 1$.

Then, for each $\mu \in M_{F}(E)$ and $T>0$,

$$
\begin{aligned}
& F\left(\mu\left(S_{T-t} Y_{t}^{(n)}\right)\right)+\int_{0}^{t} F^{\prime}\left(\mu\left(S_{T-s}\left(\frac{1}{2} Y_{s}^{(n)^{2}}\right)\right)\right) d s \\
& \quad-n \int_{0}^{t} E_{\xi^{(n)}}\left[F\left(\mu\left(S_{T-s}\left(Y_{s}^{(n)}+\frac{\xi^{(n)}}{\sqrt{n}} Y_{s}^{(n)}\right)\right)\right)-F\left(\mu\left(S_{T-s} Y_{s}^{(n)}\right)\right)\right] d s
\end{aligned}
$$

is an $\mathscr{F}_{t}^{n}$-martingale on $0 \leq t \leq T$.

Proof. From our construction of $Y^{(n)}$ it can be readily shown that in both cases

$$
F\left(\mu\left(S_{T-s}\left(Y_{s}^{(n)}+\frac{h}{\sqrt{n}} Y_{s}^{(n)}\right)\right)\right), F\left(\mu\left(S_{T-s} Y_{s}^{(n)}\right)\right) \in L_{1}^{(n)} .
$$

Hence, the result follows from Lemma 4.13 and the definition of $\hat{N}^{(n)}$ (see [11], page 62).

Next we shall obtain bounds on the moments of $Y^{(n)}$.

LEMMA 4.15. For each $T>0$ and $\mu \in M_{F}(E)$,

$$
\sup _{t \leq T} E\left[\mu\left(Y_{t}^{(n)}\right)^{3}\right]
$$

is bounded uniformly in $n$. 
Proof.

$$
\begin{aligned}
& E\left[\mu\left(S_{T-t} Y_{t}^{(n)}\right)^{3}\right] \leq \mathrm{const} \cdot \mu\left(S_{T} \phi\right)^{3} \\
& + \text { const } \cdot n E\left[\int _ { 0 } ^ { t } E _ { \xi ^ { ( n ) } } \left[\mu\left(S_{T-s}\left(Y_{s}^{(n)}+\frac{\xi^{(n)}}{\sqrt{n}} Y_{s}^{(n)}\right)\right)^{3}\right.\right. \\
& \left.\left.-\mu\left(S_{T-s} Y_{s}^{(n)}\right)^{3}\right] d s\right] \\
& E_{\xi^{(n)}}\left[\mu\left(S_{T-s}\left(Y_{s}^{(n)}+\frac{\xi^{(n)}}{\sqrt{n}} Y_{s}^{(n)}\right)\right)^{3}-\mu\left(S_{T-s} Y_{s}^{(n)}\right)^{3}\right] \\
& =E_{\xi^{(n)}}\left[n^{-3 / 2} \mu\left(S_{T-s}\left(\xi^{(n)} Y_{s}^{(n)}\right)\right)^{3}+3 n^{-1} \mu\left(S_{T-s} Y_{s}^{(n)}\right) \mu\left(S_{T-s}\left(\xi^{(n)} Y_{s}^{(n)}\right)\right)^{2}\right. \\
& \left.+3 n^{-1 / 2} \mu\left(S_{T-s} Y_{s}^{(n)}\right)^{2} \mu\left(S_{T-s}\left(\xi^{(n)} Y_{s}^{(n)}\right)\right)\right] \\
& =n^{-3 / 2} \int_{x} \int_{y} E_{z} E_{\xi^{(n)}}\left[\xi^{(n)}(x) \xi^{(n)}(y) \xi^{(n)}(z)\right] Y_{s}^{(n)}(x) Y_{s}^{(n)}(y) Y_{s}^{(n)}(z) \\
& \times S_{T-s}^{*} \mu(d x) S_{T-s}^{*} \mu(d y) S_{T-s}^{*} \mu(d z) \\
& +3 n^{-1} \mu\left(S_{T-s} Y_{s}^{(n)}\right) \int_{x} \int_{y} E_{\xi^{(n)}}\left[\xi^{(n)}(x) \xi^{(n)}(y)\right] Y_{s}^{(n)}(x) Y_{s}^{(n)}(y) \\
& \times S_{T-s}^{*} \mu(d x) S_{T-s}^{*} \mu(d y) \\
& +3 n^{-1 / 2} \mu\left(S_{T-s} Y_{s}^{(n)}\right)^{2} \int_{x} E_{\xi^{(n)}}\left[\xi^{(n)}(x)\right] Y_{s}^{(n)}(x) S_{T-s}^{*} \mu(d x) \\
& =n^{-3 / 2} \int_{x} \int_{y} \int_{x} \tilde{h}_{n}(x, y, z) Y_{s}^{(n)}(x) Y_{s}^{(n)}(y) Y_{s}^{(n)}(z) \\
& \times S_{T-s}^{*} \mu(d x) S_{T-s}^{*} \mu(d y) S_{T-s}^{*} \mu(d z) \\
& +3 n^{-1} \mu\left(S_{T-s} Y_{s}^{(n)}\right) \int_{x} \int_{y} g_{n}(x, y) Y_{s}^{(n)}(x) Y_{s}^{(n)}(y) \\
& \times S_{T-s}^{*} \mu(d x) S_{T-s}^{*} \mu(d y) \\
& \leq n^{-3 / 2}\left\|\tilde{h}_{n}\right\| \mu\left(S_{T-s} Y_{s}^{(n)}\right)^{3}+3 n^{-1}\left\|g_{n}\right\| \mu\left(S_{T-s} Y_{s}^{(n)}\right)^{3},
\end{aligned}
$$

where $S_{t}^{*}$ is the adjoint for $S_{t}$ and $\|\cdot\|$ is the supremum norm. Consequently, we get

$$
\begin{aligned}
E\left[\mu\left(S_{T-t} Y_{t}^{(n)}\right)^{3}\right] \leq & \text { const } \cdot \mu\left(S_{T} \phi\right)^{3} \\
& + \text { const } \cdot\left(n^{-1 / 2}\left\|\tilde{h}_{n}\right\|+3\left\|g_{n}\right\|\right) \int_{0}^{t} E\left[\mu\left(S_{T-s} Y_{s}^{(n)}\right)^{3}\right] d s
\end{aligned}
$$


and by Gronwall's inequality, the uniform boundedness of $\left\|\tilde{h}_{n}\right\|$ and $\left\|g_{n}\right\|$ and the fact that the last inequality is satisfied for each $0 \leq t \leq T$, we are done.

Lemma 4.15 and Hölder's inequality immediately yield the following result.

Corollary 4.16. For each $T>0$,

$$
\sup _{0 \leq t \leq T, x, y \in E} E\left[Y_{t}^{(n)}(x) Y_{t}^{(n)}(y)\right]
$$

is uniformly bounded in $n$.

We need the following lemma, which is similar to Lemma 4.4.10 of [7].

Lemma 4.17. Let $T>0$. Suppose $f(s, t)$ on $D=\{(s, t): s+t \leq T, s, t \geq 0\}$ is absolutely continuous in $s$ for each fixed $0 \leq t<T$ and absolutely continuous in $t$ for each fixed $0 \leq s<T$, and, setting $\left(f_{1}, f_{2}\right) \equiv \nabla f$, suppose that

$$
\int_{D} \int\left|f_{i}(s, t)\right| d s d t<\infty, \quad i=1,2 .
$$

Then, for almost every $t, 0 \leq t \leq T$,

$$
f(t, 0)-f(0, t)=\int_{0}^{t}\left(f_{1}(s, t-s)-f_{2}(s, t-s)\right) d s .
$$

Proof. The proof is precisely that of Lemma 4.4.10 of [7].

Lemma 4.17 provides the main tool needed for the proof of the following result.

LEMMA 4.18. If $X$ is any solution of the martingale problem for $\left(A_{1}, \nu\right)$, independent of $Y^{(n)}$, such that $\nu \in \mathscr{P}\left(M_{F}(E)\right)$ has compact support, then, for each $t \geq 0$,

$$
\begin{aligned}
& E\left[\exp \left(-X_{t}(\phi)\right)\right]-E\left[\exp \left(-X_{0}\left(Y_{t}^{(n)}\right)\right)\right] \\
& =E\left[\int_{0}^{t} \exp \left(L-X_{s}\left(Y_{t-s}^{(n)}\right)\right)\right. \\
& \quad \times\left(\frac{1}{2} \int_{E \times E} g(x, y) Y_{t-s}^{(n)}(x) Y_{t-s}^{(n)}(y) X_{s}(d x) X_{s}(d y)\right. \\
& \left.\left.-n E_{\xi^{(n)}}\left[\exp \left\{-X_{s}\left(\frac{\xi^{(n)}}{\sqrt{n}} Y_{t-s}^{(n)}\right)\right\}-1\right]\right) d s\right] .
\end{aligned}
$$


Proof. Fix $T>0$ and define three functions:

$$
h_{1}, h_{2}, f: M_{F}(E) \times \mathbb{R}_{+} \times B(E)_{+} \times \mathbb{R}_{+} \mapsto \mathbb{R}
$$

by

$$
\begin{aligned}
& h_{1}(\mu, s, \phi, t) \\
& \quad=\exp \left(-\mu\left(S_{T-t-s} \phi\right)\right)\left(\frac{1}{2} \mu\left(\left(S_{T-t-s} \phi\right)^{2}\right)\right.
\end{aligned}
$$

$$
\begin{array}{r}
+\frac{1}{2} \int_{E \times E}\left(S_{T-t-s} \phi\right)(x)\left(S_{T-t-s} \phi\right)(y) \\
\times g(x, y) \mu(d x) \mu(d y)),
\end{array}
$$

$h_{2}(\mu, s, \phi, t)$

$$
\begin{aligned}
=\exp \left(-\mu\left(S_{T-t-s} \phi\right)\right)( & \frac{1}{2} \mu\left(S_{T-t-s}\left(\phi^{2}\right)\right) \\
+ & \left.n E_{\xi^{(n)}}\left[\exp \left\{-\mu\left(S_{T-t-s}\left(\frac{\xi^{(n)}}{\sqrt{n}} \phi\right)\right)\right\}-1\right]\right), \\
f(\mu, s, \phi, t) & =\exp \left(-\mu\left(S_{T-t-s} \phi\right)\right) .
\end{aligned}
$$

First, it needs to be proved that

$$
\begin{aligned}
& E\left[f\left(X_{t}, t, Y_{0}^{(n)}, 0\right)\right]-E\left[f\left(X_{0}, 0, Y_{t}^{(n)}, t\right)\right] \\
& \quad=\int_{0}^{t} E\left[h_{1}\left(X_{s}, s, Y_{t-s}^{(n)}, t-s\right)-h_{2}\left(X_{s}, s, Y_{t-s}^{(n)}, t-s\right)\right] d s
\end{aligned}
$$

for almost every $0 \leq t \leq T$. (Since our conditions are not exactly the same as in Theorem 4.4.11 of [7], we need to do this tedious work.) The proof is based on ideas of the proof of Theorem 4.4.11 of [7] and on the previous lemma. By (4.5) and Corollary 4.14 we obtain that

$$
f\left(X_{s}, s, \phi, t\right)-\int_{0}^{s} h_{1}\left(X_{r}, r, \phi, t\right) d r
$$

is an $\mathscr{F}_{s}^{X}$-martingale on $0 \leq s \leq T-t$ for each $\phi \in B(E)_{+}, 0 \leq t \leq T$, and

$$
f\left(\mu, s, Y_{t}^{(n)}, t\right)-\int_{0}^{t} h_{2}\left(\mu, s, Y_{r}^{(n)}, r\right) d r
$$


is an $\mathscr{F}_{t}^{n}$-martingale on $0 \leq t \leq T-s$ for each $\mu \in M_{F}(E)$ and $0 \leq s \leq T$. Defining

$$
F(s, t) \equiv E\left[f\left(X_{s}, s, Y_{t}^{(n)}, t\right)\right],
$$

we have (since $Y^{(n)}$ is independent of $X$ ):

$$
\begin{aligned}
& F(s, t)=F(0, t)+\int_{0}^{s} E\left[h_{1}\left(X_{r}, r, Y_{t}^{(n)}, t\right)\right] d r, \\
& F(s, t)=F(s, 0)+\int_{0}^{t} E\left[h_{2}\left(X_{s}, s, Y_{r}^{(n)}, r\right)\right] d r
\end{aligned}
$$

for $s \geq 0, t \geq 0, s+t \leq T$. Therefore, (4.15) follows from the previous lemma.

Furthermore, we show that (4.15) is satisfied for $t=T$. Let $\left\{t_{k}\right\}$ be a sequence of points such that $t_{k} \rightarrow T$ and (4.15) is satisfied for each $t_{k}$. Defining

$$
H(t, s) \equiv h_{1}\left(X_{s}, s, Y_{t-s}^{(n)}, t-s\right)-h_{2}\left(X_{s}, s, Y_{t-s}^{(n)}, t-s\right),
$$

we get from the construction of $Y^{(n)}$ that

$$
\lim _{k \rightarrow \infty} H\left(t_{k}, s\right)=H(T, s) \quad \text { a.s. for each } s .
$$

It is easy to check that

$$
\sup _{s \leq t \leq T}|H(t, s)| \leq C_{1}(n)+C_{2}(n) \sup _{t \leq T-s}\left\|Y_{t}^{(n)}\right\| \leq C_{1}(n)+C_{2}(n)\|\phi\| 2_{T_{T_{s}}^{(n)}}^{N^{(n)}}
$$

where $C_{i}(n)$ are constants and the last inequality follows from (4.8). The expectation of $2^{N_{T-s}^{(n)}}$ is finite; hence, from the dominated convergence theorem it follows that

$$
\lim _{k \rightarrow \infty} E\left[H\left(t_{k}, s\right)\right]=E[H(T, s)] \quad \text { for each } s .
$$

Once again applying the dominated convergence theorem, we obtain

$$
\lim _{k \rightarrow \infty} \int_{0}^{T} 1_{s \leq t_{k}} E\left[H\left(t_{k}, s\right)\right] d s=\int_{0}^{T-} E[H(T, s)] d s=\int_{0}^{T} E[H(T, s)] d s .
$$

The last limit and the continuity in $t$ on the left-hand side of expression (4.15) gives us that (4.15) is satisfied for $t=T$. Recalling the definition of $f, h_{1}, h_{2}$, we are done.

The following lemma is the final step in the proof of uniqueness for $A_{1}$.

Lemma 4.19. Let $X$ be as in the previous lemma. Then

$$
\lim _{n \rightarrow \infty} E\left[\exp \left(-X_{0}\left(Y_{t}^{(n)}\right)\right)\right]=E\left[\exp \left(-X_{t}(\phi)\right)\right]
$$


Proof. From Taylor's formula it follows that

$$
\begin{aligned}
n E_{\xi^{(n)}} & {\left[\exp \left\{-X_{s}\left(Y_{t-s}^{(n)}+\frac{\xi^{(n)}}{\sqrt{n}} Y_{t-s}^{(n)}\right)\right\}-\exp \left\{-X_{s}\left(Y_{t-s}^{(n)}\right)\right\}\right] } \\
= & \exp \left(-X_{s}\left(Y_{t-s}^{(n)}\right)\right) E_{\xi^{(n)}}\left[-n^{1 / 2} X_{s}\left(\xi^{(n)} Y_{t-s}^{(n)}\right)+\frac{1}{2} X_{s}\left(\xi^{(n)} Y_{t-s}^{(n)}\right)^{2}\right] \\
& -\frac{1}{3 !} n^{-1 / 2} E_{\xi^{(n)}}\left[\exp \left(-Z^{(n)}\right) X_{s}\left(\xi^{(n)} Y_{t-s}^{(n)}\right)^{3}\right] \\
= & \frac{1}{2} \exp \left(-X_{s}\left(Y_{t-s}^{(n)}\right)\right) \int_{E \times E} g_{n}(x, y) Y_{t-s}^{(n)}(x) Y_{t-s}^{(n)}(y) X_{s}(d x) X_{s}(d y) \\
& -\frac{1}{3 !} n^{-1 / 2} E_{\xi^{(n)}}\left[\exp \left(-Z^{(n)}\right) X_{s}\left(\xi^{(n)} Y_{t-s}^{(n)}\right)^{3}\right]
\end{aligned}
$$

where $Z^{(n)}$ is some nonnegative random variable. Therefore, by the previous lemma, we obtain

$$
\begin{gathered}
\left|E\left[\exp \left(-X_{t}(\phi)\right)\right]-E\left[\exp \left(-X_{0}\left(Y_{t}^{(n)}\right)\right)\right]\right| \\
=\mid E\left[\int _ { 0 } ^ { t } \left\{\exp \left(-X_{s}\left(Y_{t-s}^{(n)}\right)\right) \frac{1}{2} \int_{E \times E}\left(g(x, y)-g_{n}(x, y)\right)\right.\right. \\
\times Y_{t-s}^{(n)}(x) Y_{t-s}^{(n)}(y) X_{s}(d x) X_{s}(d y) \\
\left.\left.+\frac{1}{3 !} n^{-1 / 2} E_{\xi^{(n)}}\left[\exp \left(-Z^{(n)}\right) X_{s}\left(\xi^{(n)} Y_{t-s}^{(n)}\right)^{3}\right]\right\} d s\right] \mid \\
\leq \int_{0}^{t} E\left[\exp \left(-X_{s}\left(Y_{t-s}^{(n)}\right)\right) \frac{1}{2} \int_{E \times E}\left|g(x, y)-g_{n}(x, y)\right|\right. \\
\left.\times Y_{t-s}^{(n)}(x) Y_{t-s}^{(n)}(y) X_{s}(d x) X_{s}(d y)\right] d s \\
+\int_{0}^{t} \frac{1}{3 !} n^{-1 / 2}\left|E\left[E_{\xi^{(n)}}\left[\exp \left(-Z^{(n)}\right) X_{s}\left(\xi^{(n)} Y_{t-s}^{(n)}\right)^{3}\right]\right]\right| d s .
\end{gathered}
$$

Using the boundedness of $\exp \left(-Z^{(n)}\right)$ and interchanging the integration with respect to $X_{s}$ and $E_{\xi^{(n)}}$, we obtain

$$
\begin{aligned}
n^{-1 / 2} \mid & E\left[E_{\xi^{(n)}}\left[\exp \left(-Z^{(n)}\right) X_{s}\left(\xi^{(n)} Y_{t-s}^{(n)}\right)^{3}\right]\right] \mid \\
& \leq n^{-1 / 2}\left\|\tilde{h}_{n}\right\| E\left[X_{s}\left(Y_{t-s}^{(n)}\right)^{3}\right] \\
& \leq C_{t-s} n^{-1 / 2} E_{X}\left[X_{s}\left(S_{t-s} \phi\right)^{3}\right] \leq \tilde{C}_{t-s} n^{-1 / 2}
\end{aligned}
$$


where $C_{t-s}, \tilde{C}_{t-s}$ are constants uniformly bounded in $s$. The second inequality follows from the independence of $X$ and $Y$ and the arguments of Lemma 4.15. The last inequality follows from Lemma 4.11. Therefore, we get that

$$
\lim _{n \rightarrow \infty} n^{-1 / 2}\left|E\left[E_{\xi^{(n)}}\left[\exp \left(-Z^{(n)}\right) X_{s}\left(\xi^{(n)} Y_{t-s}^{(n)}\right)^{3}\right]\right]\right|=0
$$

uniformly in $s$ and hence the second term in (4.18) converges to 0 .

Concerning the first term in (4.18), we obtain

$$
\begin{gathered}
\int_{0}^{t} E\left[e^{-X_{s}\left(Y_{t-s}^{(n)}\right)} \frac{1}{2} \int_{E \times E}\left|g(x, y)-g_{n}(x, y)\right| Y_{t-s}^{(n)}(x) Y_{t-s}^{(n)}(y) X_{s}(d x) X_{s}(d y)\right] d s \\
\leq \frac{1}{2} \int_{0}^{t} E_{X}\left[\int_{E \times E}\left|g(x, y)-g_{n}(x, y)\right|\right. \\
\left.\times E_{Y^{(n)}}\left[Y_{t-s}^{(n)}(x) Y_{t-s}^{(n)}(y)\right] X_{s}(d x) X_{s}(d y)\right] d s .
\end{gathered}
$$

By Corollary 4.16, $\sup _{x, y \in E} E_{Y^{(n)}}\left[Y_{t-s}^{(n)}(x) Y_{t-s}^{(n)}(y)\right]$ is uniformly bounded in $n$. Furthermore, $g_{n}$ bp-converges to $g$. Hence, by the bounded convergence theorem,

$$
\begin{aligned}
& \lim _{n \rightarrow \infty} \int_{E \times E}\left|g(x, y)-g_{n}(x, y)\right| E_{Y^{(n)}}\left[Y_{t-s}^{(n)}(x) Y_{t-s}^{(n)}(y)\right] X_{s}(d x) X_{s}(d y)=0 \\
& \text { a.s. } \forall s \leq t \text {. }
\end{aligned}
$$

Since $E\left[X_{s}(1)^{2}\right]<\infty$ for each $s$, it follows from the dominated convergence theorem that

$$
\begin{aligned}
\lim _{n \rightarrow \infty} E\left[\int_{E \times E}\left|g(x, y)-g_{n}(x, y)\right| E_{Y^{(n)}}\left[Y_{t-s}^{(n)}(x) Y_{t-s}^{(n)}(y)\right] X_{s}(d x) X_{s}(d y)\right] & =0 \\
\forall s & \leq t .
\end{aligned}
$$

Once again using the bounded convergence theorem, we have

$$
\begin{aligned}
\lim _{n \rightarrow \infty} \int_{0}^{t} E[ & \int_{E \times E}\left|g(x, y)-g_{n}(x, y)\right| \\
& \left.\times E_{Y^{(n)}}\left[Y_{t-s}^{(n)}(x) Y_{t-s}^{(n)}(y)\right] X_{s}(d x) X_{s}(d y)\right] d s=0 .
\end{aligned}
$$

By (4.18), (4.19), and (4.20) we are done.

The previous lemma and Theorem 1.3 now imply the following result.

THEOREM 4.20. For each $\nu \in \mathscr{P}\left(M_{F}(E)\right)$ the martingale problem for $\left(A_{1}, \nu\right)$ has a unique solution.

REMARK 4.21. The proof of Theorem 3.1 is now complete. 
Acknowledgments. This paper is part of my Ph.D. thesis written under the supervision of Professor R. Adler. I am grateful to him for his guidance and numerous helpful conversations during the preparation of this work. I also would like to thank a referee for comments and suggestions which improved the exposition.

\section{REFERENCES}

[1] Carmona, R. and Molchanov, S. (1994). Parabolic Anderson problem and intermittency. Mem. Amer. Math. Soc. 518.

[2] Dawson, D. (1980). Spatially homogeneous random evolutions. J. Multivariate Anal. 10 141-180.

[3] Dawson, D. (1993). Measure-valued processes. École d'Été de Probabilitiés de Saint Flour. Lecture Notes in Math. 1541 1-26. Springer, New York.

[4] Dawson, D. and Fleischmann, K. (1990). Critical branching in a highly fluctuating random medium. Probab. Theory Related Fields 90 241-274.

[5] Dawson, D. and Perkins, E. (1991). Historical processes. Mem. Amer. Math. Soc. 454.

[6] Dynkin, E. (1993). Superprocesses and partial differential equations. Ann. Probab. 21 $1184-1262$.

[7] EthieR, S. N. and KuRtz, T. G. (1986). Markov Processes: Characterization and Convergence. Wiley, New York.

[8] Fleischmann, K. and Molchanov, S. (1990). Exact asymptotics in a mean field model with random potential. Probab. Theory Related Fields 86 239-251.

[9] Gartner, J. and Molchanov, S. (1990). Parabolic problems for the Anderson model. Comm. Math. Phys. 132 613-655.

[10] Helland, I. (1981). Minimal conditions for weak convergence to a diffusion process on the line. Ann. Probab. 9 429-452.

[11] Ikeda, N. and Watanabe, S. (1989). Stochastic Differential Equations and Diffusion Processes. North-Holland, Amsterdam.

[12] JACOD, J. and ShIRYAEv, A. (1987). Limit Theorems for Stochastic Processes. Springer, New York.

[13] KeIding, N. (1975). Extinction and exponential growth in random environments. Theoret. Population Biol. 8 49-63.

[14] KurTz, T. G. (1981). Approximation of Population Processes. SIAM, Philadelphia.

[15] Meleard, S. and Roelly, S. (1993). Interacting measure branching processes. Some bounds for the support. Stochastics and Stochastics Reports 44 103-121.

[16] Mueller, C. and Perkins, E. (1992). The compact support property for solutions to the heat equation with noise. Probab. Theory Related Fields 93 325-358.

[17] Perkins, E. (1992). Measure-valued branching diffusions with spatial interactions. Probab. Theory Related Fields 94 189-245.

[18] Perkins, E. (1995). On the martingale problem for interactive measure-valued branching diffusions. Mem. Amer. Math. Soc. 549.

[19] Roelly-Coppoletta, S. (1989). A criterion of convergence of measure-valued processes: application to measure branching processes. Stochastics 17 43-65.

[20] Samorodnitsky, G. and TAqqu, M. (1994). Stable Non-Gaussian Random Processes. Chapman and Hall, New York.

[21] WaLsh, J. (1986). An introduction to stochastic partial differential equations. Lecture Notes in Math. 1180 265-439. Springer, New York.

FACUlty of Industrial EngineERING ANd Management

TeChNion-IsRaEl Institute of TeChNOLOGY

HAIFA 32000

ISRAEL

E-MAIL: leonid@ie.technion.ac.il 